\title{
Bisphosphonate-related osteonecrosis. Application of adipose-derived stem cells in an experimental murine model
}

Estefanía Alonso-Rodriguez ${ }^{1}$, Javier González-Martín-Moro ${ }^{1}$, José-Luis Cebrián-Carretero ${ }^{1}$, José-Luis Del Castillo ${ }^{2}$, Jose-Juan Pozo-Kreilinger ${ }^{3}$, Elena Ruiz-Bravo ${ }^{3}$, Mariano García-Arranz ${ }^{4,5}$, Juan Hernández-Godoy ${ }^{6}$, Miguel Burgueño ${ }^{2}$

${ }^{1}$ M.D. Department of Oral and Maxillofacial Surgery, Hospital Universitario La Paz, Paseo de la Castellana 261, 28046 Madrid, Spain. Chief of Department: Miguel Burgueño

${ }^{2}$ M.D, Ph.D. Department of Oral and Maxillofacial Surgery, Hospital Universitario La Paz, Paseo de la Castellana 261,28046 Madrid, Spain. Chief of Department: Miguel Burgueño

${ }^{3}$ M.D. Department of Pathology, Hospital Universitario La Paz, Paseo de la Castellana 261, 28046 Madrid, Spain

${ }^{4}$ Department of Surgery, Faculty of Medicine, Universidad Autónoma de Madrid, C/ Arzobispo Morcillo s/n, 28029 Madrid, Spain

${ }^{5}$ Cell Therapy Laboratory, Instituto de Investigación Sanitario Fundación Jiménez Díaz, Avda. Reyes Católicos 2,28040 Madrid, Spain

${ }^{6}$ M.D. Plastic Surgery. Private practice

Correspondence:

Paseo de la Castellana, 261

28046 Madrid, Spain

Department of Oral and Maxillofacial Surgery

stfania@hotmail.com

Received: 01/02/2019 Accepted: 13/03/2019

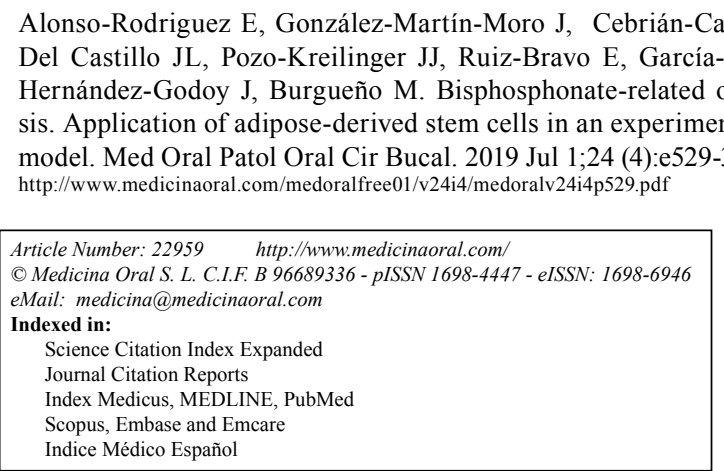

\begin{abstract}
Background: Bisphosphonate-related osteonecrosis of the jaw is a pathological condition without effective established treatment and preventive strategies. The aim of this study was to analyse the effect of adipose-derived stem cells (ASC) in an experimental murine model of osteonecrosis.

Material and Methods: 38 Wistar rats were injected intraperitoneally with zoledronic acid. After treatment, upper jaw molars were extracted. The animals were randomly assigned to one of two groups. In the control group, saline solution was applied over the alveolar sockets after the tooth extractions. In the treatment group, ASCs were applied instead of saline solution. The control and treatment groups were subdivided based on the time of euthanasia. A clinical and histological analysis was performed.

Results: The presence of osteonecrosis in alveolar bone was observed in a similar distribution in both groups. In the ASC-treated group, new bone formation was greater than in controls.
\end{abstract}


Conclusions: In this study, application of ASCs showed greater new bone formation in an osteonecrosis-like murine model. Previous inhibited post-extraction bone remodelling could be reactivated, and these findings appeared to be secondary to implantation of ASCs.

Key words: Osteonecrosis, bisphosphonates, mesenchymal stem cells (MSC), adipose-derived stem cells (ASCs), zoledronic acid.

\section{Introduction}

Medication-related osteonecrosis of the jaw (MRONJ) is a painful condition that severely affects quality of life. The main drugs associated with MRONJ are bisphosphonates (BPs), which are antiresorptive drugs used to treat many diseases and conditions of increased bone resorption such as osteoporosis, Paget's disease, multiple myeloma, hypercalcaemia of malignancy or osteolytic bone metastases, among others.

Osteonecrosis of the jaw is a pathological condition of increasing frequency with a poorly understood pathophysiology. Despite all of the studies and advances achieved, MRONJ still lacks effective treatment and preventive strategies. The main hypotheses regarding the pathophysiology of osteonecrosis are pharmacological inhibition of bone remodelling and angiogenesis, the presence of trauma and/or inflammation/infection, soft tissue BP toxicity, and an innate or acquired immune dysfunction $(1,2)$. Given the variety of hypotheses, there are many treatments but none is totally successful. Due to the lack of curative treatment for MRONJ, one of the best strategies nowadays is to avoid it.

Mesenchymal stem cells (MSC) have recently been studied for the treatment of MRONJ, and they appear to be beneficial for this issue. However, there are few published studies. Some are case reports and most use MSCs isolated from bone marrow (BM-MSC) (3-9). Studies on prevention of MRONJ and stem cells are emerging $(4,10,11)$ but they are scarce, and this field needs to be investigated.

Adipose tissue is an abundant and easily accessible source of MSCs, known as adipose-derived stem cells (ASCs). The capacity of ASCs to promote angiogenesis, secrete growth factors, regulate the inflammatory process, and differentiate into multiple cell types makes them a potentially ideal therapy for chronic wounds (12). These properties make ASCs very attractive as a possible tool for prevention of MRONJ.

The aim of this study was to analyse the potential effect of ASCs in an experimental murine model of BRONJ (bisphosphonate-related osteonecrosis of the jaw)-like disease induced with zoledronic acid (ZA) after tooth extraction without other enhancer drugs.

\section{Material and Methods}

-Study design

Currently, there is no standard MRONJ animal model.
A previously published MRONJ-like disease model(13) has been followed with some variations. The present study was approved by the Ethics Committee for Animal Care of La Paz University Hospital, Madrid, Spain (CEI 69-1212-A131).

Thirty-eight Wistar female and two male rats (Charles River, France) with an average age of 8 weeks and an average weight of 275 grams were used. All the animals were kept in a controlled-temperature environment with food and water ad libitum.

All the female animals were injected intraperitoneally with zoledronic acid (ZA) (Sandoz, $4 \mathrm{mg} / 100 \mathrm{ml}$ ) at dosages of $0.1 \mathrm{mg} \mathrm{ZA} / \mathrm{kg}$ three times per week for 9 weeks. The males received no treatment, they were used just to obtain adipose tissue. After this treatment, three right upper molars were extracted from all the animals under general anaesthesia with the objective of establishing the osteonecrosis model.

The molars were extracted with under optical microscope assistance. After syndesmotomy with a spatula, the molars were luxated with a spatulated osteotrimmer and a mosquito clamp was used as forceps (Fig. 1A). Surgical milling of the alveolar bone was carried out with a tungsten bur without irrigation to add more trauma.

The animals were randomly assigned to one of two groups. In the control group, saline solution was applied over the alveolar sockets after the tooth extractions. In the treatment group, ASCs were applied instead of saline solution (one million allogeneic cells in 25 microlitres of Ringer's lactate solution in each maxilla). An absorbable haemostatic gelatine sponge (Spongostan ${ }^{\circledR}$ ) was used as a carrier for ASCs or for saline solution in each group (Fig. 1B). An advanced buccal mucoperiosteal flap to cover the alveolar bone post-extraction was performed for all the animals. Gingival borders were attached with a continuous suture covering the defect without tension (Fig. 1C).

After tooth extractions, the animals were decapitated under general anaesthesia (inhaled isoflurane with oxygen $3 \mathrm{~L} / \mathrm{min}$ ) and the heads were harvested for histological study. The control and treatment groups were subdivided as follows based on the time of euthanasia:

- Group 1: 6 control rats, euthanised 1 month after tooth extraction;

- Group 2: 8 treatments rats, euthanised 1 month after tooth extraction; 


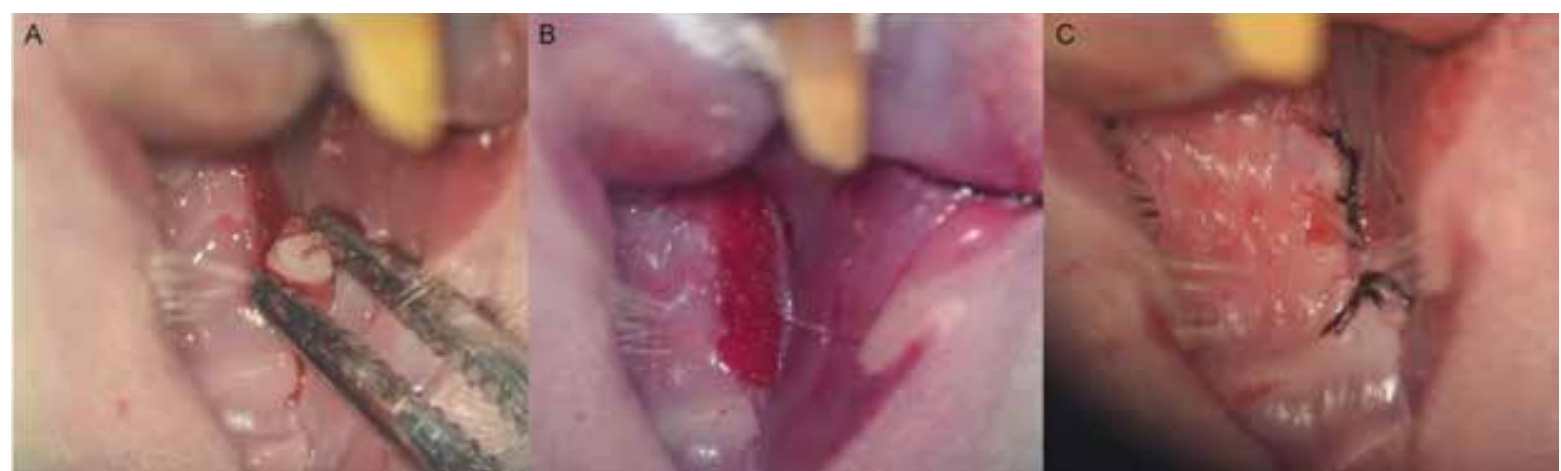

Fig. 1: A: Tooth extraction with optical microscope assistance; B: haemostatic sponge with ASCs over the alveolar sockets; C: advanced buccal mucoperiosteal flap.

- Group 3: 6 control rats, euthanised 2 months after tooth extraction;

- Group 4: 9 treatments rats, euthanised 2 months after tooth extraction.

Tramadol was administered once preoperatively, and daily subcutaneously for 3 postoperative days.

Intraperitoneal general anaesthesia (ketamine $100 \mathrm{mg} /$ $\mathrm{kg}+$ diazepam $8 \mathrm{mg} / \mathrm{kg}+$ atropine $0.4 \mathrm{mg} / \mathrm{kg}$ ) was first used; however, 9 rats (all controls) died during the procedure or on the postoperative days. Isoflurane was then used with no further deaths until euthanasia.

-ASC preparation

Allogeneic ASCs were obtained under inhaled general anaesthesia from the subcutaneous abdominal and inguinal fat tissue of the two male Wistar rats. This process is based on separation by centrifugation, use of type I collagenase and the plastic adhesion properties of the cells.

Fat tissue was digested with type I collagenase $(0.075 \%$; Gibco BRL, Paisley, Scotland, UK). The collagenase was then inactivated by addition of an equal volume of Dulbecco's Modified Eagle Medium (DMEM; Gibco BRL), which contained $10 \%$ foetal bovine serum (FBS; Gibco BRL). The digested tissue was centrifuged at 300 $\mathrm{G}$ for $10 \mathrm{~min}$. After filtering through a $70-\mu \mathrm{m}$ nylon mesh, the cells were cultured in DMEM (Gibco BRL) containing 10\% FBS (Gibco BRL) and 1\% penicillinstreptomycin (Gibco BRL). The medium was changed to remove nonadherent cells $24 \mathrm{~h}$ after seeding and every 3-4 days thereafter. When $80 \%-90 \%$ confluence was achieved, the cells were detached with $0.05 \%(\mathrm{v} / \mathrm{v})$ trypsin-ethylenediaminetetraacetic acid (Gibco BRL) in phosphate-buffered saline. The subcultured cells were frozen until the week before their use.

-Histological analysis

Histological features are not included in the American Association of Oral and Maxillofacial Surgeons' definition of MRONJ in humans. However, in rats, this description is one of the main tools for studying osteonecrosis. In this study, the characteristics of osteonecrosis of the jaw described previously in the literature have been followed $(14,15)$. Histological sections were stained with haematoxylin-eosin, periodic acid-Schiff and Masson's trichrome and the analysis was performed by two pathologists in a blinded fashion (light microscopy, 40x magnification). The parameters evaluated were as follows:

1. Osteonecrosis: defined as a portion of bone in which greater than or equal to 10 adjacent empty osteocyte lacunae are present, as previously described by Yamashita et al. (16), in 3 different random cuts (20x).

2. Osteoclast count: Arithmetic mean of the count in three different fields (40x), in two different random cuts in the alveolar process of the upper jaw.

3. Vascularisation: graded by a visual scale from 1 to 5 (1: absence of vessels; 5 : duplication of the vascular surface area compared with this area in the controls).

4. Degree of alveolar remodelling (after tooth extraction): A scale was established from 0 to 4 ( 0 : absence of bone resorption; $1:<25 \% ; 2: 25-50 \% ; 3: 50-75 \%$; 4 : $75-100 \%$ of alveolar bone area resorbed and substituted by fibrous tissue).

5. Bone neoformation: New bone formation graded on a scale from 0 to 4 ( 0 : absence of bone formation; $1:<$ $25 \%$; $2: 25-50 \%$; $3: 50-75 \%$; $4: 75-100 \%$ of bone formation).

6. Inflammatory infiltrates: Evaluated as presence or absence.

-Statistical analysis

The data analyses were performed with SPSS v. 20. A statistical significance level of $p<.05$ was used.

The quantitative variables were analysed with the nonparametric Kruskal-Wallis test or the Mann-Whitney $\mathrm{U}$ test if necessary.

The qualitative variables were analysed with the $\chi^{2}$ test or Fisher's exact test (if $\mathrm{N}<20$ or if any expected value is less than 5). When the $\chi^{2}$ test was used, Yates's correction was applied.

\section{Results}

None of the animals had exposed bone at the extraction sites. 
-ASCs versus controls

When all the ASC-treated rats (groups 2 and 4) and all the control rats (groups 1 and 3) were compared (Table 1), all showed the presence of osteonecrosis in alveolar bone in a similar distribution.

In the ASC-treated group, new bone formation was statistically significantly greater (with a mean value of 3.00 in the treatment group vs. 2.18 in the control group; $p=0.044$ ) (Figs. 2-4).
Vascularisation showed a greater mean value in the ASC-treated rats compared with the control group without reaching statistical significance.

No differences were observed in the remaining variables (inflammatory infiltrates, osteoclast count and bone remodelling).

-ASC vs. control rats euthanised 1 month after tooth extraction

Differences in groups 1 and 2 (treated and control rats

Table 1: ASC versus control rats results.

\begin{tabular}{|l|c|c|c|}
\hline & Group & Mean value & $p$ \\
\hline Osteonecrosis & Control & $63.6 \%(7 / 11)$ & 1 \\
& ASCs & $66.7 \%(10 / 15)$ & \\
\hline Vascularisation & Control & 2.18 & 0.243 \\
& ASCs & 2.40 & \\
\hline Bone remodeling & Control & 2.18 & 0.619 \\
& ASCs & 2.27 & \\
\hline Inflammatory infiltrates & Control & $72.7 \%(8 / 11)$ & 0.428 \\
& ASCs & $53.3 \%(8 / 15)$ & \\
\hline Osteoclasts count & Control & 7.45 & 0.044 \\
& ASCs & 7.20 & \\
\hline Bone neoformation & Control & 2.18 & \\
& ASCs & 3.00 & \\
\hline
\end{tabular}

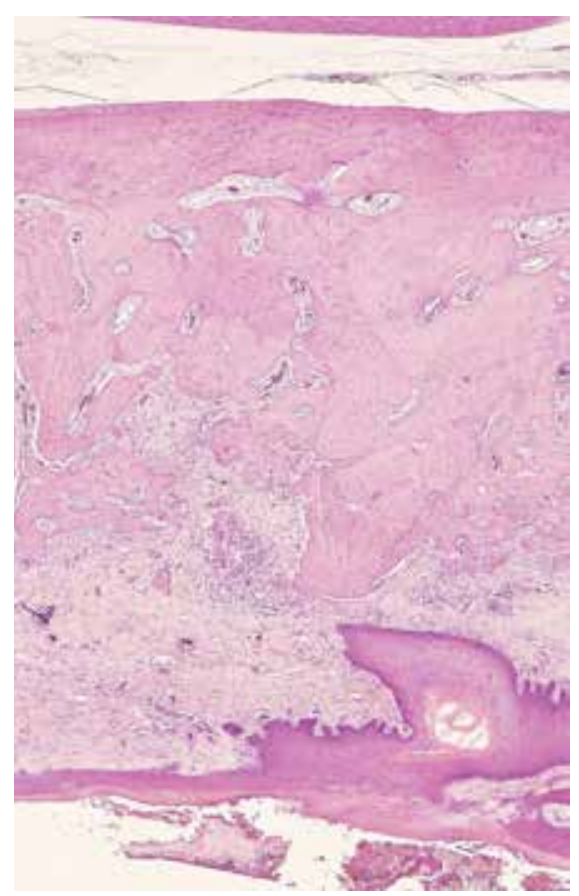

Fig. 2: Histologic image of extraction sockets (haematoxylin-eosin, 4x). Control specimen (group 1). Little bone neoformation is observed.

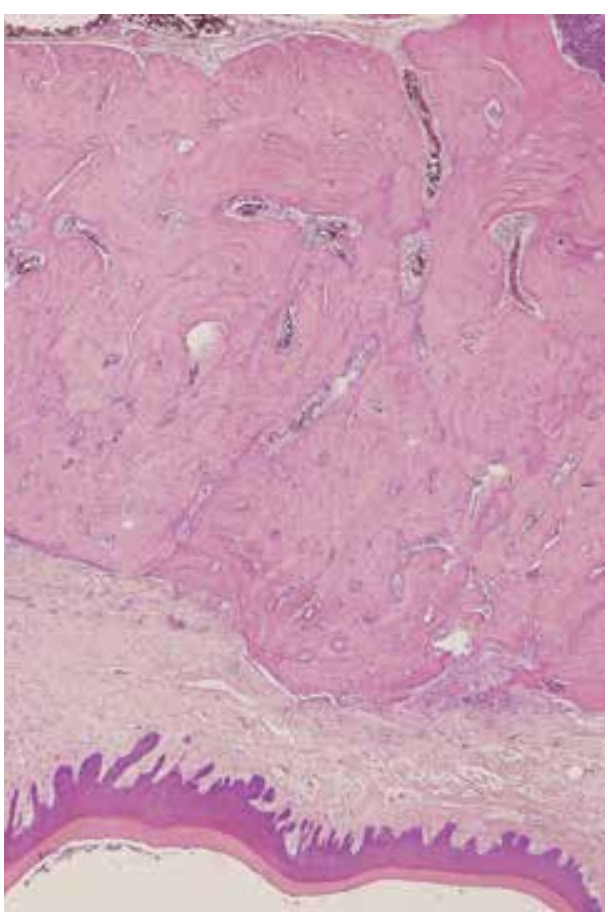

Fig. 3: Histologic image of extraction sockets (haematoxylin-eosin, 4x). ASC-treated specimen (group 2). Greater new bone formation is observed in the treated specimen. 


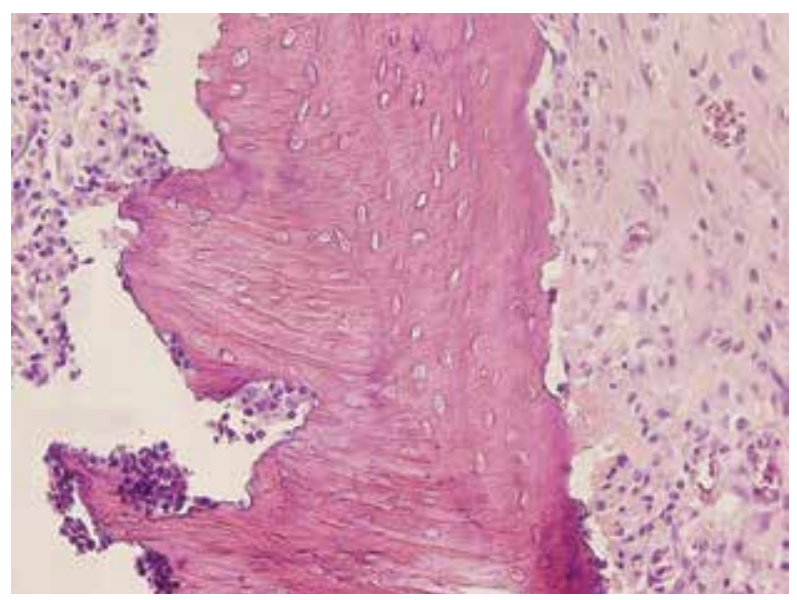

Fig. 4: Histologic image of osteonecrosis (haematoxylin-eosin, 40x). Control specimen (group 1). Empty osteocyte lacunae are observed.

euthanised 1 month after dental extractions) were analysed (Table 2).

Vascularisation and bone formation had greater mean values in the ASC-treated rats than in the control group, though statistical significance was not reached.

No statistically significant differences were found in osteonecrosis, osteoclasts, bone remodelling or inflammatory infiltrates.

-ASC vs. control rats euthanised 2 months after dental extraction

Vascularisation and bone formation showed greater mean values in the ASC-treated rats versus the control group, though without reaching statistical significance (Table 3).

Osteoclast numbers were greater in group 4 than in group 3, with a tendency towards statistical significance.
No statistically significant differences were found in osteonecrosis, bone remodelling or inflammatory infiltrates.

\section{Discussion}

Currently, there is no standard BRONJ animal model due to a high degree heterogeneity of models (BP type, dosage, concomitant treatments...). In this study, osteonecrosis was induced after chronic administration of ZA, based on previous results(13) without other drugs to evaluate the real effect of BP treatment, with tooth extraction being the trigger for BRONJ-like lesions. As prevention strategies are essential in MRONJ, the potential preventive effect of locally applied ASCs on bone remodelling and regeneration of tooth extraction sockets has been evaluated.

None of the animals showed macroscopic bone exposure at the extraction sites. It is important to note that the unexposed bone stage of MRONJ (stage 0 ) in the AAOMS classification for humans, defined as clinical and radiological findings without bone exposure, nowadays appears to be a recognized stage (1). Despite the absence of bone exposure, histological features of osteonecrosis have been observed. When all the ASC-treated rats and all the control rats were compared, the presence of empty lacunae in alveolar bone was observed in a similar distribution. Osteonecrosis is similarly in both groups (in $66.7 \%$ of the specimens in the ASC group and $63.3 \%$ in the control group). Other authors such as Kobayashi et al. (17) didn't observe bone exposure either. In their murine model, $250 \mu \mathrm{g} / \mathrm{Kg} /$ day of ZA was injected from 7 days before tooth extraction to 4 days after extraction. Although no macroscopic changes were observed in Kobayashi's model, new bone formation in the tooth

Table 2: ASC versus control rats euthanised 1 month after tooth extraction.

\begin{tabular}{|c|c|c|c|}
\hline & Group & Mean value & $p$ \\
\hline Osteonecrosis & $\begin{array}{l}\text { Control } \\
\text { ASCs }\end{array}$ & $\begin{array}{l}83.3 \%(5 / 6) \\
66.7 \%(4 / 6)\end{array}$ & 1 \\
\hline Vascularisation & $\begin{array}{c}\text { Control } \\
\text { ASCs }\end{array}$ & $\begin{array}{c}2 \\
2.17\end{array}$ & .317 \\
\hline Bone remodeling & $\begin{array}{l}\text { Control } \\
\text { ASCs }\end{array}$ & $\begin{array}{c}2 \\
2.17\end{array}$ & .317 \\
\hline Inflammatory infiltrates & $\begin{array}{c}\text { Control } \\
\text { ASCs }\end{array}$ & $\begin{array}{l}66.7 \%(4 / 6) \\
66.7 \%(4 / 6)\end{array}$ & 1 \\
\hline Osteoclasts count & $\begin{array}{l}\text { Control } \\
\text { ASCs }\end{array}$ & $\begin{array}{l}8.33 \\
5.83\end{array}$ & .259 \\
\hline Bone neoformation & $\begin{array}{c}\text { Control } \\
\text { ASCs }\end{array}$ & $\begin{array}{l}2.17 \\
2.83\end{array}$ & .356 \\
\hline
\end{tabular}


Table 3: ASC versus control rats euthanised 2 months after tooth extraction.

\begin{tabular}{|l|c|c|c|}
\hline & Group & Mean value & $p$ \\
\hline Osteonecrosis & Control & $40 \%(2 / 5)$ & .580 \\
& ASCs & $66.7 \%(6 / 9)$ & \\
\hline Vascularisation & Control & 2.40 & .591 \\
& ASCs & 2.56 & \\
\hline Bone remodeling & Control & 2.40 & .810 \\
& ASCs & 2.33 & .301 \\
\hline Inflammatory infiltrates & Control & $80 \%(4 / 5)$ & .079 \\
& ASCs & $44.4 \%(4 / 9)$ & \\
\hline Osteoclasts count & Control & 6.40 & .113 \\
& ASCs & 8.11 & \\
\hline Bone neoformation & Control & 2.20 & \\
& ASCs & 3.14 & \\
\hline
\end{tabular}

extraction socket was suppressed in mice treated with ZA. Matsumoto et al. (18) observed a deficiency of bone formation and remodelling but no bone exposure in aged rats with a more prolonged but inferior dose of ZA $(0.035 \mathrm{mg} / \mathrm{kg}$ every 15 days).

Alveolar coverage with a mucoperiosteal flap, surgical milling of alveolar bone, the high regeneration capacity of the rat gingiva and the lack of other osteonecrosis enhancer drugs could have contributed to the absence of macroscopic bone exposure.

It was necessary to cover the alveolar sockets with a mucoperiosteal flap to contain the cells, and this step could have promoted healing, prevented contamination and decreased the risk of bone exposure. Lody et al. (19) and Heufelder et al. (20) recommend this measure in their protocols to prevent development of BRONJ after tooth extraction. Surgical milling of alveolar bone was carried out with a tungsten bur with the intention of adding more trauma. This manoeuvre possibly acted as an alveolectomy. It has been suggested that alveolectomy might favour complete healing of the extraction wound and decrease the risk of the occurrence of MRONJ(21).

In many BRONJ-like murine models, open wounds with bone exposure is only observed when BP is combined with corticoids $(22,23)$ or chemotherapeutic therapy(11). In the present study, only ZA was administered to avoid confounding factors in the outcomes.

BPs inhibit the action of osteoclasts and induce apoptosis. This process leads to decreased bone resorption and remodelling. When osteoclastic action does not occur, the necessary steps for the activation of osteoblasts does not take place (24). An important finding in this study is that in the ASC-treated group, new bone formation was greater and statistically significant (see Table 1) and was always greater in the ASC subgroups independent of the time of evolution after tooth extraction. This discovery is important because it could be attributed to the effect of stem cells.

No differences were found in the number of osteoclasts when comparing both groups. However, the treated rats euthanised 2 months after tooth extraction showed a greater mean value than the control group, with a tendency towards statistical significance. This difference could also be due to the effect of the stem cells applied. The increase in bone regeneration and bone remodelling could be attributed to the capacity of ASCs to differentiate directly into multiple mesodermal cell types (25), as well as into cells of ectodermal and endodermal lineages $(26,27)$. However, according to findings in the literature, it is more probable that these results were related to the paracrine effects induced by ASCs. Lee et al. (28) have shown that ASCs could express and secret various cytokines, growth factors and proteins that are required for bone function and remodelling like $\mathrm{M}$ CSF, RANKL, BMP-2, BMP-4, HGF.

Inhibition of angiogenesis is one of the hypotheses on the pathophysiology of osteonecrosis. ZA has shown significant antiangiogenic activity in several in vitro and in vivo models through a reduction in serum VEGF (Vascular endothelial growth factor) levels (29,30). In addition, it has been demonstrated that ASCs secrete multiple proangiogenic growth factors as VEGF(31). Vascularisation showed a greater mean value in the ASC-treated rats in all groups, though statistical significance was not reached.

Adipose tissue has been used in this model because it is an abundant and easily accessible source of stem cells. In addition, the procedure to obtain ASCs is a safe and minimally invasive procedure, associated with low discomfort for patients. Although BM-MSCs and ASCs share many properties, they also present some differ- 
ences. Some studies suggest that BM-MSCs could be more prone to osteogenic differentiation than ASCs in vitro $(32,33)$, but other authors don't find any differences $(34,35)$. ASCs seems to have a higher immunomodulatory capacity than BM-MSCs $(36,37)$ which could improve immune dysfunction related to BPs $(4,6)$.

There are few reports about stem cells and MRONJ and most of them study the treatment effects but not the preventive effects of ASCs.

Some studies on MSCs in the treatment of this condition are the reports of Kaibuchi et al. (3), Kikuiri et al. (4) and Ogata et al. (5), which have shown that treatment with BM-MSCs is effective for the treatment of osteonecrosis-like disease in murine models. ZA and dexamethasone had been administered in these reports to generate the BRONJ-like model. Li et al. (6) observed bone exposure with BP-only treatment in larger animals (minipigs), and showed new bone formation in previously necrotic areas after BM-MSC infusion. There are a few reports on humans with good results, but with a low level of evidence (7-9). All of these studies use MSCs isolated from bone marrow.

Stem cells have been recently investigated as a preventive factor in MRONJ. Kikuiri et al. (4) showed that systemic infusion of BM-MSCs prevents and cures BRONJ-like disease in a mouse model via induction of peripheral tolerance. Kuroshima et al. (11) investigated the effects of non-cultured stromal vascular fraction (SFV) transplantation on tooth extraction socket healing in mice. Specimens were treated previously with chemotherapeutic/bisphosphonate combination therapy and they stated that systemic transplantation of SVF cells ameliorates BRONJ-like lesions by improving both osseous and soft tissue healing of tooth extraction sockets. The SVF is a heterogenous population including endothelial cells, pericytes, fibroblasts, preadipocytes, ASCs... $(25,38)$, so they have different properties compared with purified ASCs.

With the objective of eliminating possible confounding factors and analysing only the effects of ASCs in the present study, stimulation with bone morphogenetic protein 2 and platelet-rich plasma have been eliminated from our research group's previous model (10). In the present model using ASCs only, new bone formation was greater in the treated group, suggesting that previously inhibited post-extraction bone remodelling could be reactivated. There is also bone formation in the control group, but it is significantly less and since the only difference between groups is the implantation of ASCs, these findings appeared to be secondary to the effects of stem cells.

Further studies are needed to confirm our preliminary results on ASCs as a preventive strategy in MRONJ, but these results appear to be promising.

Prevention strategies are essential in MRONJ and this field needs to be investigated. In this study, the effect of ASCs in an experimental murine model of osteonecrosis was analysed and the treated group has shown greater new bone formation. These results are promising and establish a basis for further studies and subsequent clinical trials in humans on the effect of ASCs as a preventive factor for this pathology.

\section{References}

1. Ruggiero SL, Dodson TB, Fantasia J, Goodday R, Aghaloo T, Mehrotra B, et al. American association of oral and maxillofacial surgeons position paper on medication-related osteonecrosis of the jaw - 2014 update. J Oral Maxillofac Surg. American Association of Oral and Maxillofacial Surgeons. 2014;72:1938-56.

2. Aghaloo T, Hazboun R, Tetradis S. Pathophysiology of Osteonecrosis of the Jaws. Oral Maxillofac Surg Clin North Am 2015: 27(4): 489-496. doi: 10.1016/j.coms.2015.06.001

3. Kaibuchi N, Iwata T, Yamato M, Okano T, Ando T. Multipotent mesenchymal stromal cell sheet therapy for bisphosphonate-related osteonecrosis of the jaw in a rat model. Acta Biomater. 2016;42:40010.

4. Kikuiri T, Kim I, Yamaza T, Akiyama K, Zhang Q, Li Y, et al. Cell-based immunotherapy with mesenchymal stem cells cures bisphosphonate-related osteonecrosis of the jaw-like disease in mice. J Bone Min Res. 2010;25: 1668-79.

5. Ogata K, Katagiri W, Osugi M, Kawai T, Sugimura Y, Hibi H, et al. Evaluation of the therapeutic effects of conditioned media from mesenchymal stem cells in a rat bisphosphonate-related osteonecrosis of the jaw-like model. Bone. 2015;74: 95-105.

6. Li Y, Xu J, Mao L, Liu Y, Gao R, Zheng Z, et al. Allogeneic Mesenchymal Stem Cell Therapy for Bisphosphonate-Related Jaw Osteonecrosis in Swine. Stem Cells Dev. 2013;22: 2047-56.

7. Gonzálvez-García M, Rodríguez-Lozano FJ, Villanueva V, Segarra-Fenoll D, Rodríguez-González MA, Oñate-Sánchez R, et al. Cell therapy in bisphosphonate-related osteonecrosis of the jaw. J Craniofac Surg. 2013;24: e226-8.

8. Cella L, Oppici A, Arbasi M, Moretto M, Piepoli M, Vallisa D, et al. Autologous bone marrow stem cell intralesional transplantation repairing bisphosphonate related osteonecrosis of the jaw. Head Face Med. 2011;17:16.

9. Voss PJ, Matsumoto A, Alvarado E, Schmelzeisen R, Duttenhöfer F, Poxleitner P. Treatment of stage II medication-related osteonecrosis of the jaw with necrosectomy and autologous bone marrow mesenchymal stem cells. 2017;105: 484-93.

10. Barba-Recreo P, Del Castillo Pardo de Vera JL, Georgiev-Hristov T, Ruiz Bravo-Burguillos E, Abarrategi A, Burgueño M, et al. Adipose-derived stem cells and platelet-rich plasma for preventive treatment of bisphosphonate-related osteonecrosis of the jaw in a murine model. J Craniomaxillofac Surg. 2015;43:1161-8.

11. Kuroshima S, Sasaki M, Nakajima K, Tamaki S, Hayano H, Sawase T. Transplantation of Noncultured Stromal Vascular Fraction Cells of Adipose Tissue Ameliorates Osteonecrosis of the Jaw-Like Lesions in Mice. J Bone Min Res. 2018;33:154-66.

12. Toyserkani NM, Christensen ML, Sheikh SP, Sørensen JA. Adipose-Derived Stem Cells: New treatment for wound healing. Ann Plast Surg. 2015; 75:117-23.

13. Barba-recreo P, Del Castillo Pardo de Vera JL, García-arranz M, Yébenes L, et al. Zoledronic acid - Related osteonecrosis of the jaws. Experimental model with dental extractions in rats. J Craniomaxillofac Surg. 2014;42:744-50

14. Fantasia JE. Bisphosphonates-What the Dentist Needs to Know: Practical Considerations. J Oral Maxillofac Surg. 2009;67:53-60.

15. Favia G, Pilolli GP, Maiorano E. Histologic and histomorphometric features of bisphosphonate-related osteonecrosis of the jaws: An analysis of 31 cases with confocal laser scanning microscopy. Bone. 2009;45:406-13

16. Yamashita J, Koi K, Yang D-Y, McCauley LK. Effect of Zoledro- 
nate on Oral Wound Healing in Rats. Clin Cancer Res. 2011;17:140514.

17. Kobayashi Y, Hiraga T, Ueda A, Wang L, Matsumoto-Nakano M, Hata $\mathrm{K}$, et al. Zoledronic acid delays wound healing of the tooth extraction socket, inhibits oral epithelial cell migration, and promotes proliferation and adhesion to hydroxyapatite of oral bacteria, without causing osteonecrosis of the jaw, in mice. J Bone Min Metab. 2010;28:165-75.

18. Matsumoto MA, de Abreu Furquim EM, Gonçalves A, SantiagoJúnior JF, Saraiva PP, Cardoso CL, et al. Aged rats under zoledronic acid therapy and oral surgery. J Craniomaxillofac Surg. 2017;45:7817.

19. Lodi G, Sardella A, Salis A, Demarosi F, Tarozzi M, Carrassi A. Tooth Extraction in Patients Taking Intravenous Bisphosphonates: A Preventive Protocol and Case Series. J Oral Maxillofac Surg. 2010;68:107-10.

20. Heufelder MJ, Hendricks J, Remmerbach T, Frerich B, Hemprich A, Wilde F. Principles of oral surgery for prevention of bisphosphonate-related osteonecrosis of the jaw. Oral Surg Oral Med Oral pathol Oral Radiol. 2014;117: e429-35.

21. Gaudin E, Seidel L, Bacevic M, Rompen E, Lambert F. Occurrence and risk indicators of medication-related osteonecrosis of the jaw after dental extraction: a systematic review and meta-analysis. J Clin Periodontol. 2015;42: 922-32.

22. López-Jornet P, Camacho-Alonso F, Molina-Miñano F, GómezGarcía F, Vicente-Ortega V. An experimental study of bisphosphonate-induced jaws osteonecrosis in Sprague-Dawley rats. J Oral Pathol Med. 2010;39: 697-702.

23. Abtahi J, Agholme F, Sandberg O, Aspenberg P. Bisphosphonateinduced osteonecrosis of the jaw in a rat model arises first after the bone has become exposed. No primary necrosis in unexposed bone. J Oral Pathol Med. 2012;41:494-9.

24. Marx RE, Sawatari Y, Fortin M, Broumand V. Bisphosphonateinduced exposed bone (osteonecrosis/osteopetrosis) of the jaws: Risk factors, recognition, prevention, and treatment. J Oral Maxillofac Surg. 2005;63:1567-75.

25. Zuk PA, Zhu M, Mizuno H, Huang J, Futrell JW, Katz AJ, et al. Multilineage Cells from Human Adipose Tissue: Implications for Cell-Based Therapies. 2001; 7: 211-8.

26. Baer PC. Adipose-Derived Stem Cells and Their Potential to Differentiate into the Epithelial Lineage. Stem Cells Dev. 2011;20:180516.

27. Ferroni L, Gardin C, Tocco I, Epis R, Casadei A, Vindigni V, et al. Potential for Neural Differentiation of Mesenchymal Stem Cells. Adv Biochem Eng Biotechnol. 2013;129: 89-115.

28. Lee K, Kim H, Kim JM, Kim JR, Kim KJ, Kim YJ, et al. Systemic transplantation of human adipose-derived stem cells stimulates bone repair by promoting osteoblast and osteoclast function. J Cell Mol Med. 2011;15:2082-94.

29. Wood J, Bonjean K, Ruetz S, Bellahcène A, Devy L, Foidart JM, et al. Novel antiangiogenic effects of the bisphosphonate compound zoledronic acid. J Pharmacol Exp Ther. 2002;302:1055-61.

30. Santini D, Vincenzi B, Dicuonzo G, Avvisati G, Massacesi C, Battistoni F, et al. Advances in Brief Zoledronic Acid Induces Significant and Long-Lasting Modifications of Circulating Angiogenic Factors in Cancer Patients. Clin Cancer Res. 2003;9:2893-7

31. Rehman J, Traktuev D, Li J, Merfeld-Clauss S, Temm-Grove CJ, Bovenkerk JE, et al. Secretion of Angiogenic and Antiapoptotic Factors by Human Adipose Stromal Cells. Circulation. 2004;109:1292-8. 32. Bochev I, Elmadjian G, Kyurkchiev D, Tzvetanov L, Altankova I, Tivchev P, et al. Mesenchymal stem cells from human bone marrow or adipose tissue differently modulate mitogen-stimulated B-cell immunoglobulin production in vitro. Cell Biol Int. 2008;32:384-93.

33. Noël D, Caton D, Roche S, Bony C, Lehmann S, Casteilla L, et al. Cell specific differences between human adipose-derived and mesenchymal-stromal cells despite similar differentiation potentials. Exp Cell Res. 2008;314:1575-84.

34. De Ugarte DA, Morizono K, Elbarbary A, Alfonso Z, Zuk PA, Zhu M, et al. Comparison of Multi-Lineage Cells from Human Adi- pose Tissue and Bone Marrow. Cells Tissues Organs. 2003;174: 1019.

35. Pachón-Peña G, Yu G, Tucker A, Wu X, Vendrell J, Bunnell BA, et al. Stromal stem cells from adipose tissue and bone marrow of agematched female donors display distinct immunophenotypic profiles. J Cell Physiol. 2011;226: 843-51.

36. Melief SM, Zwaginga JJ, Fibbe WE, Roelofs H. Adipose TissueDerived Multipotent Stromal Cells Have a Higher Immunomodulatory Capacity Than Their Bone Marrow-Derived Counterparts. Stem Cells Transl Med. 2013;2: 455-63.

37. Li C, Wu X, Tong J, Yang X, Zhao J, Zheng Q, et al. Comparative analysis of human mesenchymal stem cells from bone marrow and adipose tissue under xeno-free conditions for cell therapy. Stem Cell Res Ther. 2015;6:55.

38. Peinado JR, Pardo M, de la Rosa O, Malagón MM. Proteomic characterization of adipose tissue constituents, a necessary step for understanding adipose tissue complexity. Proteomics. 2012;12:60720.

\section{Conflicts of interest}

MGA is a co-author of 2 patents entitled "Identification and isolation of multipotent cells from non-osteochondral mesenchymal tissue" (10157355957US) and "Use of adipose tissue-derived stromal stem cells in treating fistula" (US11/167061). The Universidad Autónoma de Madrid (UAM) and Cellerix share the patent rights.

This study was supported by a grant from the Fondo de Investigación Sanitaria (FIS), Spain.

The remaining authors have no other financial or competing interests to declare. 